\title{
Preoperative Inflammation Markers in Predicting Biochemical Recurrence after Robot-assisted Radical Prostatectomy
}

\author{
Mahmut Taha Olcucu, Kaan Karamik, Kayhan Yilmaz, Cagatay Ozsoy, Yasin Aktas and Mutlu Ates \\ Department of Urology, University of Health Sciences, Antalya Training and Research Hospital, Turkey
}

\begin{abstract}
Objective: To evaluate the importance of preoperative neutrophil-to-lymphocyte ratio (NLR), lymphocyte-to-monocyte ratio (LMR), platelet-to-lymphocyte ratio (PLR), and neutrophil-to-monocyte ratio (NMR) in prostate cancer patients, who underwent robot-assisted radical prostatectomy (RARP).

Study Design: Observational study.

Place and Duration of Study: Urology Department, Antalya Training and Research Hospital, Turkey, between March 2015 and September 2019.

Methodology: Four hundred and fifty-four patients underwent RARP were scanned. Clinical characteristics and pathological features of patients were recorded. Patients were excluded, if they had persistent PSA; a history of any autoimmune or inflammatory disease; anti-inflammatory agents use; blood transfusion within 3 months; or a follow-up time shorter than 3 months. Systemic inflammation markers were calculated and correlated with patients' data and biochemical recurrence (BCR). Biochemical recurrence was defined as two repetitive measurements of PSA levels $\geq 0.2 \mathrm{ng} / \mathrm{mL}$ at 3 months after the radical prostatectomy. Mann-Whitney U-test, Fisher's exact test, and Pearson Chi-square test, ROC curve, Kaplan-Meier survival analyses, and Cox proportional hazard regression model were used as statistical methods.

Results: Four hundred and two patients were eligible. The median age at RP was 65.5 (61-69) years and median PSA of the patients was $8.3(5.76-12.61) \mathrm{ng} / \mathrm{ml}$. Median NLR, LMR, PLR, and NMR were 2 (1.55-2.61), 3.86 (3.14-5), 105.69 (85-134.29), 7.82 (6.25-9.71); and optimal cut-off values were $2.33,3.75,106.6$, and 8.75, respectively. Low LMR was found as an important predictor of biochemical recurrence (hazard ratio, $\mathrm{HR}=1.769,95 \%$ confidence interval, $\mathrm{Cl}=1.091-2.868, \mathrm{p}=0.021$ ). A significant association was found between lower LMR and decreased BCR -free survival $(p<0.001)$.

Conclusion: Pretreatment low LMR might be a simple and inexpensive index, which reflects the host systemic immunity and can predict independently BCR after RARP.
\end{abstract}

Key Words: Biochemical recurrence, Lymphocyte-to-monocyte ratio, Neutrophil-to-lymphocyte ratio, Neutrophil-to-monocyte ratio, Platelet-to-lymphocyte ratio, Prostate cancer.

How to cite this article: Olcucu MT, Karamik K, Yilmaz K, Ozsoy C, Aktas Y, Ates M. Preoperative Inflammation Markers in Predicting Biochemical Recurrence after Robot-assisted Radical Prostatectomy. J Coll Physicians Surg Pak 2020; 30(09):921-927.

\section{INTRODUCTION}

Prostate cancer (PCa) is the most common solid cancer type in men. ${ }^{1}$ The diagnosis of PCa has increased with screening measurement of prostate-specificantigen (PSA). ${ }^{2}$ Radical prostatectomy $(\mathrm{RP})$ is an effective treatment option in prostate cancer.

Correspondence to: Dr. Mahmut Taha Olcucu, Department of Urology, University of Health Sciences, Antalya Training and Research Hospital, Turkey

E-mail: matah ol@hotmail.com

Received: June 04, 2020; Revised: September 18, 2020;

Accepted: September 30, 2020

DOI: https://doi.org/10.29271/jcpsp.2020.09.921
Compared to external beam radiotherapy, RP demonstrates equivalent oncological outcomes and also provides tumor control, accurate staging and elimination of possible PSA sources. However, biochemical recurrence (BCR) may occur in $35 \%$ of patients, after which it is usually associated with bad prognosis. ${ }^{3}$

Biochemical recurrence is known as with two repetitive measurements of PSA levels $\geq 0.2 \mathrm{ng} / \mathrm{mL}$ at 3 months after the RP. ${ }^{4}$ Persistent PSA is described as PSA levels $\geq 0.1 \mathrm{ng} / \mathrm{mL}$ within 6 weeks after the RP; and it is associated with invisible metastases at the time of surgery. ${ }^{4}$ Patients who experience BCR after RP, which may require secondary therapy, have poorer oncological outcomes. Several clinical and pathological factors have been proven to be independent predictors of BCR after surgery. ${ }^{5}$ Nevertheless, the predictors of $B C R$ remain unclear. Hence, effective prognostic biomarkers are needed for individualised risk assessment and clinical decision-making. 


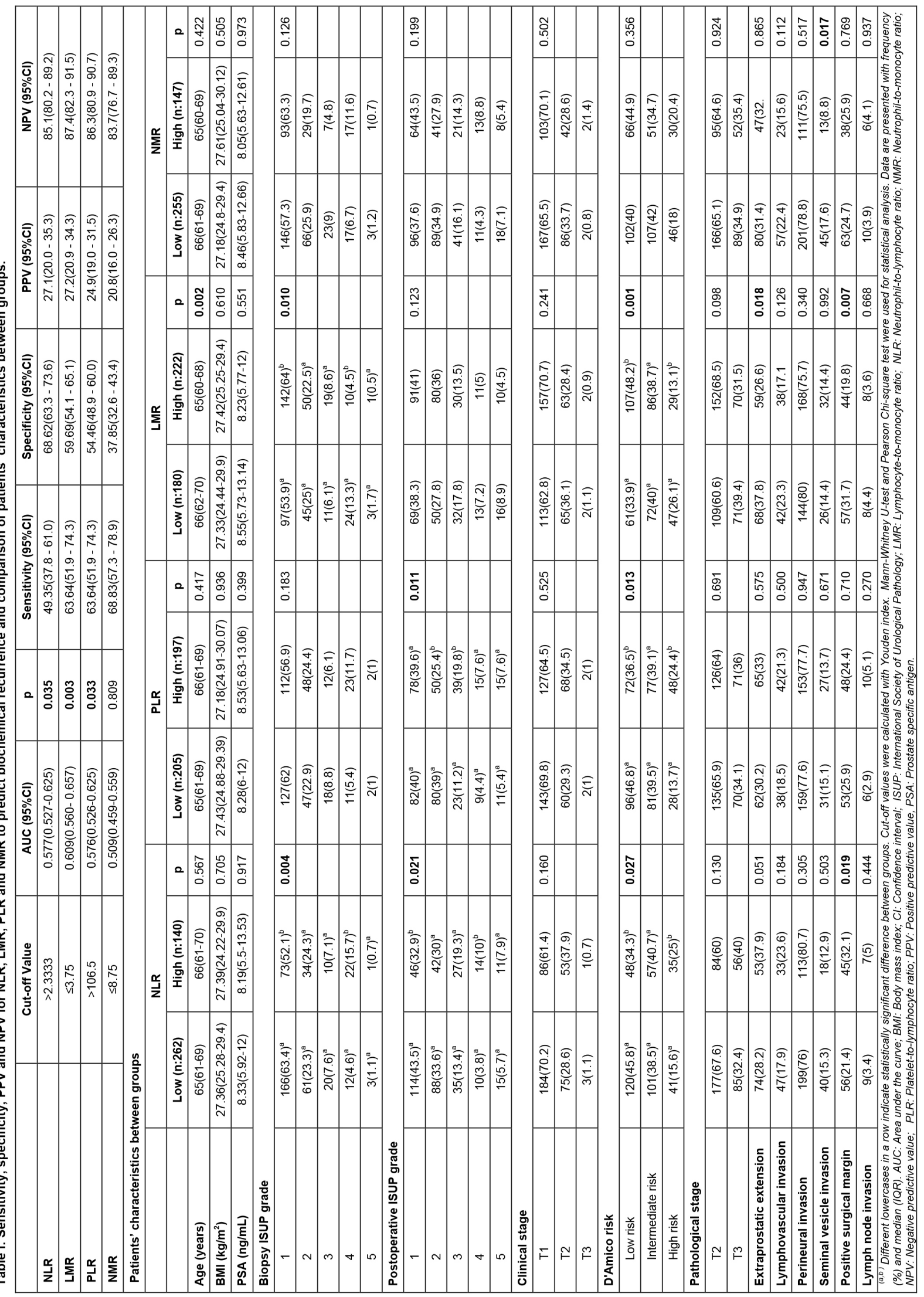


Tumor-associated inflammation is considered as a key factor for cancer development and progression. ${ }^{6}$ The ratio of neutrophil-to-lymphocyte (NLR), neutrophil-to-monocyte (NMR), lymphocyte-to-monocyte (LMR), platelet-to-lymphocyte (PLR) can be calculated from complete blood counts $(C B C)$; and these have been suggested as an emerging marker of systemic inflammation. The body's response to inflammation has a critical role in patients with malignancies. ${ }^{7,8}$ Consequently, the NLR has been evaluated for predicting $\mathrm{BCR}$ in $\mathrm{PCa}$; however, results of respective studies are controversial. ${ }^{9-11}$

In cancer patients, the inflammation markers are increasingly important in terms of prognosis. Moreover, the inflammation markers are an accessible and inexpensive clinical parameters in daily practice.

The aim of the present study was to evaluate the inflammation markers in predicting biochemical recurrence in prostate cancer patients who underwent robot-assisted RP (RARP).

\section{METHODOLOGY}

This observational study was conducted at the Urology Department, Antalya Training and Research Hospital. After Local Ethics Committee approval, the data of 454 patients who had underwent robot-assisted radical prostatectomy without neo-adjuvant treatment between March 2015 and September 2019 at the Urology Department of Antalya Training and Research Hospital were retrospectively identified. Patients were excluded, if they had PSA levels $\geq 0.1$ $\mathrm{ng} / \mathrm{mL}$ within 6 weeks after RP (persistent PSA); a history of any autoimmune or inflammatory disease; anti-inflammatory drug use; blood transfusion within 3 months; or a follow-up time shorter than 3 months.

Da Vinci Xi Robotic System ${ }^{\circledR}$ (Intuitive Surgical System Technologies, Sunnyvale, CA, USA) was used to perform surgeries. Patients with a lymph node metastasis risk of above $5 \%$ according to the Briganti nomogram had extended lymph node dissection. Clinical characteristics and pathological features of patients including age, preoperative PSA, biopsy of International Society of Urological Pathology (ISUP) grade, clinical T stage, postoperative ISUP grade, pathological T stage, surgical margin (SM), lymphovascular invasion (LVI), extraprostatic extension (EPE), presence of perineural invasion (PNI), seminal vesicle invasion (SVI) and presence of lymph node metastasis were noted. The CBCs was obtained 3-10 days before surgery. The NLR, PLR, LMR and NMR were computed and noted. After the RARP, the patients were followed up postoperatively with PSA. BCR presence was defined as two repetitive measurements of PSA levels $\geq 0.2 \mathrm{ng} / \mathrm{mL}$ at 3 months after the RP. $B C R$-free survival (BCRFS) was calculated from the time of RP to BCR.
SPSS (version 23.0, IBM Corp., Armonk, NY) and MedCalc Statistical Software (Unlincenced copy-free trial, version 19.3.1, MedCalc Software Ltd, Ostend, Belgium) were used for statistical analysis. Qualitative data were given as frequency (\%), while quantitative as median (IQR). The normality of patients' data was controlled by the ShapiroWilk test. Mann-Whitney U-test was used to determine the differences between the groups. The Fisher's exact test and Pearson Chi-square test were used for categorical variables. The receiver operating characteristic (ROC) curve analysis was applied to evaluate the predictive performance of $C B C$ parameters for BCR. The area under the curve (AUC), sensitivity, specificity, negative and positive predictive values (NPV-PPV) were computed and found with $95 \%$ confidence intervals. Youden's index was used to find the optimal cutoff points. The Kaplan-Meier analysis was used to demonstrate survival curves; and the log-rank test was used to evaluate the differences. Univariate and multivariate analyses of independent predictors of BCR was performed with a Cox proportional hazard regression model. The variables which showed significant association with BCR in the univariate analyses were further tested in a backward stepwise multivariate model. Hazard ratio (HR), with corresponding 95\% confidence intervals $(95 \% \mathrm{Cls})$, was reported. $\mathrm{P}<0.05$ was accepted as significant.

\section{RESULTS}

Four hundred and two patients were found eligible for the study. The entire cohort aged 65.5 (61-69) years on median (IQR) and median PSA of the patients were 8.3 (5.76-12.61) $\mathrm{ng} / \mathrm{ml}$. Median NLR, LMR, PLR, and NMR were 2 (1.55-2.61), 3.86 (3.14-5), 105.69 (85-134.29), and 7.82 (6.25-9.71), respectively. After RARP, patients were followed-up with a median of 19 months.

Based on the ROC curve and Youden's index, the potential NLR cut-off point was 2.33 for BCR (AUC $=0.577$ ). The value of 3.75 for LMR ( $A \cup C=0.609$ ) and 106.5 for PLR (AUC $=0.576$ ) were found to be a cut-off point. Finally, a cutoff point of 8.75 was calculated for NMR (AUC $=0.509$ ). Table I reports the sensitivity, specificity, PPV, and NPV for study parameters to predict BCR. According to cut-off points, low and high groups were created (Table I). High-NLR group had a higher biopsy and postoperative ISUP grade and D'Amico risk classification than those in the low-NLR group ( $p=0.004$, $p=0.021$, and $p=0.027$, respectively). Furthermore, patients with NLR $>2.33$ were more likely to harbour positive SMs $(p=0.019)$. The high-PLR group was correlated with higher postoperative ISUP grade and D'Amico risk classification

$(p=0.011$ and $p=0.013)$. The high-LMR group was slightly younger than the low-LMR group $(p=0.002)$. D'Amico risk classification and biopsy ISUP grade of the low-LMR group were significantly higher than the high-LMR group. Additionally, the low-LMR group had more EPE and positive SM than the high-LMR group ( $p=0.018$ and $p=0.007)$. 
Table II: Comparison of patients' characteristics between BCR groups and demonstration of univariate and backward stepwise multivariate Cox regression analysis for determining the risk factors associated with biochemical reccurence.

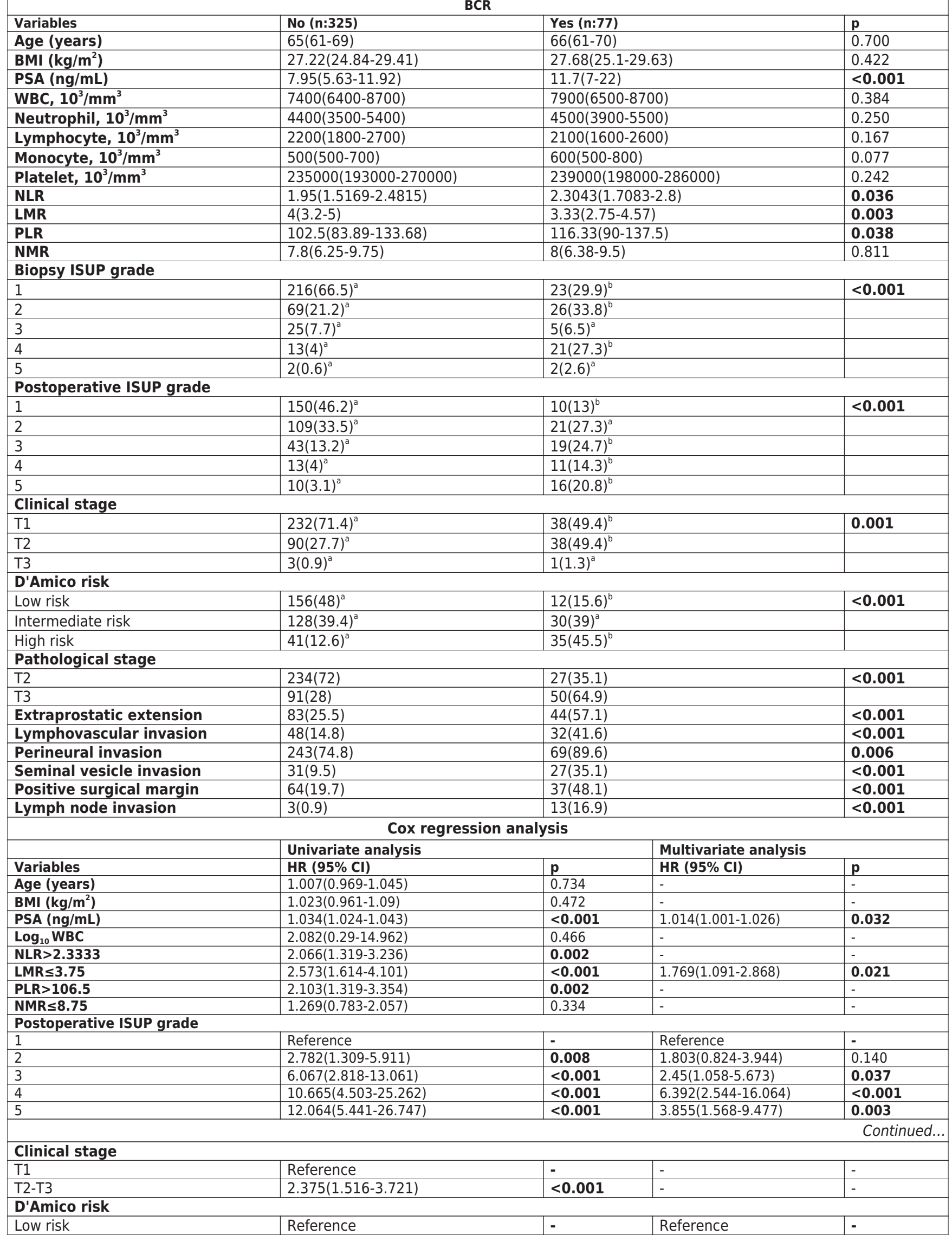




\begin{tabular}{|c|c|c|c|c|}
\hline Intermediate risk & $3.244(1.657-6.348)$ & 0.001 & $2.389(1.168-4.886)$ & 0.017 \\
\hline High risk & $9.46(4.885-18.321)$ & $<0.001$ & $2.731(1.248-5.978)$ & 0.012 \\
\hline $\mathrm{T} 2$ & Reference & - & - & - \\
\hline Extraprostatic extension & $3.307(2.104-5.198)$ & $<0.001$ & - & - \\
\hline Lymphovascular invasion & $3.26(2.07-5.132)$ & $<0.001$ & - & - \\
\hline Perineural invasion & $2.389(1.147-4.976)$ & 0.020 & - & - \\
\hline Lymph node invasion & $11.912(6.402-22.165)$ & $<0.001$ & $3.672(1.737-7.761)$ & 0.001 \\
\hline \multicolumn{5}{|c|}{$\begin{array}{l}\text { (a, b) Different lowercases in a row indicate statistically significant difference between groups. Mann-Whitney U-test, Pearson Chi-square test and } \\
\text { Fisher's Exact test were used for statistical analysis. Data are presented with frequency (\%) and median (IQR). BMI: Body mass index; Cl: } \\
\text { Confidence interval; HR: Hazard ratio; ISUP: International Society of Urological Pathology; LMR: Lymphocyte-to-monocyte ratio, NLR: Neutrophil- } \\
\text { to-lymphocyte ratio; NMR: Neutrophil-to-monocyte ratio; PLR: Platelet-to-lymphocyte ratio; PSA: Prostate specific antigen; WBC: White blood cell. }\end{array}$} \\
\hline
\end{tabular}

The relationship of $B C R$ with study parameters after RP is shown in Table II. PSA, NLR, LMR, PLR, biopsy and postoperative ISUP grade, clinical stage, D'Amico risk, pathological stage, positive SM, EPE, LVI, PNI, SVI, and lymph node invasion were found to be statistically significant. The independent predictors of BCR were shown in Table II. BCR was independently associated with PSA (hazard ratio, $H R=1.014$, $p=0.032)$, positive $S M(H R=2.302, p=0.001)$, and lymph node invasion $(H R=3.672, p=0.001)$. Only $L M R(H R=1.769$, $p=0.021$ ) remained to be a significant predictor of $B C R$ among inflammation markers.
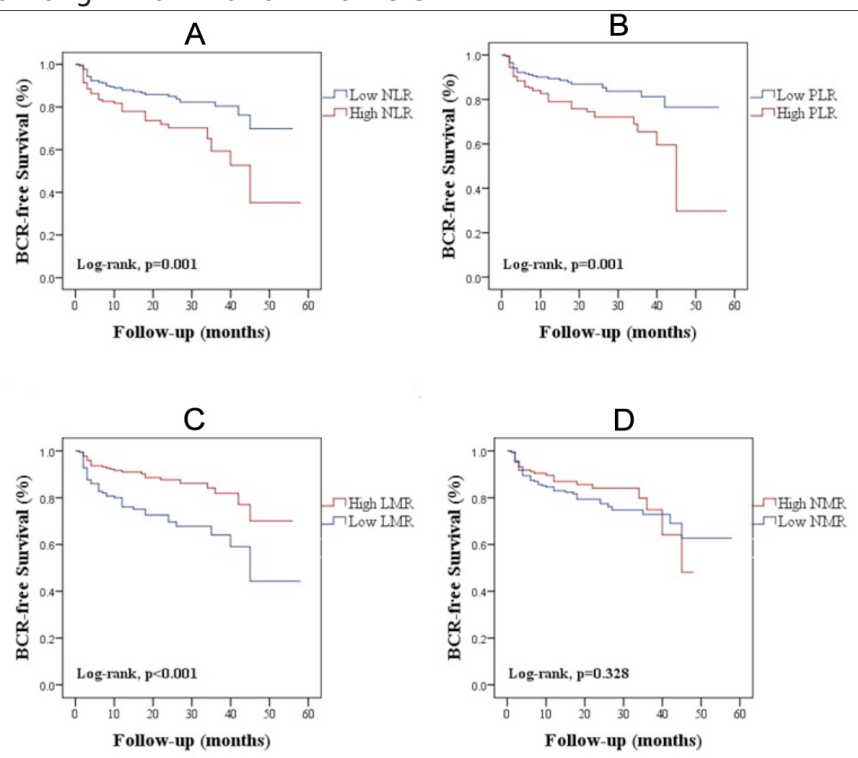

Figure 1: Biochemical recurrence-free survival curves according to predictive factors. The high-NLR (A), high-PLR (B), low-LMR (C) were significantly associated with decreased BCR-free survival. No association was found between NMR and BCR-free survival (D). BCR: Biochemical recurrence; LMR: Lymphocyte-to-monocyte ratio; NLR: Neutrophil-to-lymphocyte ratio; NMR: Neutrophil-to-monocyte ratio; PLR: Platelet-to-lymphocyte ratio.

BCR developed in 77 patients; and the mean time between $\mathrm{RP}$ and $\mathrm{BCR}$ was $18.09 \pm 12.49$. Kaplan-Meier survival analyses of patients are presented in Figure 1. The BCRFS was significantly shorter in the high-NLR group $(p=0.001)$ (Figure $1 A)$. The high PLR was significantly associated with decreased BCRFS $(p=0.001)$ (Figure 1B). A significantly higher risk of experiencing BCR was found in the low-LMR group $(p<0.001)$ (Figure $1 C)$. In terms of BCRFS, low and high NMR groups were not significantly different $(p=0.328)$ (Figure 1D).

\section{DISCUSSION}

The present study demonstrated that preoperative low LMR might be an independent factor in prediction of BCR after RARP. After scanning literature, no study was found to demonstrate an association between LMR and BCR. This study is the first to demonstrate lower LMR was associated with higher $B C R$ rates. It is also found here that increased NLR and PLR, decreased LMR were associated with poor BCRFS.

Previous studies reported the association between the inflammation markers and the clinical outcome of the malignancies. ${ }^{7,12}$ However, the exact mechanisms underlying the prognostic capacity of systemic inflammation markers remain to be clarified. The effects of neutrophils on the proliferation of tumor cells are well known. ${ }^{13}$ Lymphocytes have a major role in inhibiting cancer cell proliferation and migration. ${ }^{14}$ They have an antitumor effect. The decreased lymphocyte counts are correlated with an immunosuppressive status. Monocytes might differentiate into tumor-associated macrophages (TAM) when necessary, and TAM might support tumor growth, invasion, metastasis and angiogenesis. ${ }^{15}$ Therefore, the number of monocytes could be representative of TAMs reflecting the tumor burden. The body's inflammatory response to malignancy is lymphocyte dependent, and there is an association between the high level of TAM and tumor invasiveness and clinical outcomes. Thus, LMR could reflect the inflammation states in the tumor microenvironment. Because of a reduction in lymphocytes or an increase in monocytes, low LMR would be associated with unfavourable oncological outcomes in cancer patients. CBC tests are frequently used in routine practice and LMR is an accessible and inexpensive clinical parameter. LMR is a costeffective parameter that can be used in clinical practice to estimate the outcomes of cancer patients.

There is increasing evidence correlating the lower LMR with poorer oncological results in patients with several malignan- 
cies. ${ }^{16-18}$ This study demonstrated that LMR is an independent factor to estimate BCR after RARP. Nishijima et al. performed a systematic review and meta-analysis including the patients with non-hematologic solid tumors. A low LMR represented an unfavorable factor for clinical outcomes. ${ }^{19} \mathrm{Li}$ et al. also published a meta-analysis to demonstrate the importance of LMR in the urologic malignancies. ${ }^{20}$ However, there was no PCa patient in the studies including this meta-analysis. In a study including 214 castration-resistant PCa patients, absolute monocyte count (AMC) and LMR had associated with bad prognosis according to univariate analysis. ${ }^{21}$ However, AMC remained an independent prognostic factor for prognosis.

Several meta-analyses have been performed to further validate the prognostic importance of NLR in PCa. ${ }^{22,23}$ Tang et al. reported that NLR could be a marker in predicting the outcomes of locally advanced and/or castration-resistant PCa patients. ${ }^{22} \mathrm{~A}$ recent systematic review also demonstrated that high NLR was associated with bad prognosis in all of the stratified categories except localised $\mathrm{PCa} .{ }^{23}$ The importance of NLR in PCa is not yet clear. High NLR had a significant association with $\mathrm{BCR}$ in univariate analysis, which was found in the present study.

BCR may occur in 35\% after RP. ${ }^{3}$ Detecting BCR after RP is important in identifying treatment failure and considering salvage therapy. Several clinical and pathological findings, such as PSA, stage of the disease, high Gleason score (GS), and positive surgical margin (PSM) are well-known predictors of BCR. ${ }^{5,24}$ In this study, PSA, high GS, PSM, and lymph node invasion were independently predicted BCR. The authors had previously described the importance of the De Ritis ratio in predicting BCR after $\mathrm{RP},{ }^{25}$ and found that it was an independent prognostic factor for prediction of BCR.

The mean time between radical prostatectomy and BCR was 18.09 months in the present study, which is relatively short when compared with the literature. RARP technique has a learning curve to gain experience. Initial RARP cases were included in this study, which were in the learning curve. This may cause shorter BCR mean time after RARP.

Most of the studies on this subject are retrospective. Therefore, there might be a bias to select patients. Large-scale prospective studies are needed to assess the presence of biomarkers to predict BCR after RP.

The present study also has some limitations. It has a retrospective design and the data are obtained from a single institution. Second, the population was also small with a short follow-up duration. Third, the role of other various medical conditions such as smoking, metabolic syndrome, cardiovascular diseases and some other unknown factors that could affect the results, was not evaluated in multivariate analyses.

\section{CONCLUSION}

Preoperative low LMR might be an independent predictor for $B C R$ in the patients who underwent RARP, which is a simple and inexpensive method. Low LMR was also more likely to have poor BCRFS. The prognostic utility of LMR should be evaluated in further large prospective cohorts.

\section{ETHICAL APPROVAL:}

Ethics Committee approval was received for this study from the Ethics Committee of Antalya Training and Research Hospital (Approval No. 4/10 - 03.2020).

\section{PATIENTS' CONSENT:}

Data of this retrospective study was collected from clinical archive.

\section{CONFLICT OF INTEREST:}

Authors declared no conflict of interest.

\section{AUTHORS' CONTRIBUTION:}

MTO: Conception, materials, data collection, analysis, writing and critical review.

KK: Conception, materials and writing.

$\mathrm{KY}$ : Materials and critical review.

CO: Data collection.

YA: Data collection.

MA: Material and critical review.

\section{REFERENCES}

1. Segal R, Miller K, Jemal A. Cancer statistics, 2018. CA Cancer J Clin 2018; 68(1):7-30. doi: 10.3322/caac. 21442.

2. Masood A, Iqbal N, Shohab D, Hassan A, Aimon S, Mehmood $U$, et al. Clinicopathological characteristics of prostate cancer in patients presenting to a tertiary care private sector hospital. J Coll Physicians Surg Pak 2018;28:409-11. doi: 10.29271/jcpsp.2018.05.409.

3. Han M, Partin AW, Pound CR, Epstein JI, Walsh PC. Longterm biochemical disease-free and cancer-specific survival following anatomic radical retropubic prostatectomy. The 15-year Johns Hopkins experience. Urol Clin North Am 2001; 28(3):555-565. doi: 10.1016/s0094-0143(05)70163-4.

4. Preisser F, Chun FKH, Pompe RS, Heinze A, Salomon G, Graefen $M$, et al. Persistent prostate-specific antigen after radical prostatectomy and its impact on oncologic outcomes. Eur Urol 2019; 76(1):106-114. doi: 10.1016/j.eururo.2019.01.048.

5. Kotb AF, Elabbady AA. Prognostic factors for the development of biochemical recurrence after radical prostatectomy. Prostate Cancer 2011; 2011:485189. doi: 10.1155/2011/ 485189 .

6. Coussens LM, Werb Z. Inflammation and cancer. Nature 2002; 420(6917):860-7. doi: 10.1038/nature01322.

7. Wu G, Yao Y, Bai C, Zeng J, Shi D, Gu X, et al. Combination of platelet to lymphocyte ratio and neutrophil to lymphocyte ratio is a useful prognostic factor in advanced non-small cell lung cancer patients. Thorac Cancer 2015; 6(3):275-87. doi: 10.1111/1759-7714.12178. 
8. An X, Ding PR, Li YH, Wang FH, Shi YX, Zang ZQ, et al. Elevated neutrophil to lymphocyte ratio predicts survival in advanced pancreatic cancer. Biomarkers 2010; 15(6):516-22. doi: 10.3109/1354750X.2010.491557.

9. Minardi D, Scartozzi M, Montesi L, Santoni M, Burattini L, Bianconi $M$, et al. Neutrophil-to-lymphocyte ratio may be associated with the outcome in patients with prostate cancer. Springerplus 2015; 4:255. doi: 10.1186/s40064015-1036-1.

10. Lu Y, Huang HH, Lau WKO. Evaluation of neutrophil-to-lymphocyte ratio as a prognostic indicator in a Singapore cohort of patients with clinically localized prostate cancer treated with prostatectomy. World J Urol 2020; 38(1):103-9. doi: 10.1007/s00345-019-02752-4.

11. Zhang GM, Zhu Y, Ma XC, Qin XJ, Wan FN, Dai B, et al. Pretreatment neutrophil-to-lymphocyte ratio: A predictor of advanced prostate cancer and biochemical recurrence in patients receiving radical prostatectomy. Medicine (Baltimore) 2015; 94(41):e1473. doi: 10.1097/MD.00000000 00001473.

12. Shimada H, Takiguchi $N$, Kainuma O, Soda H, Ikeda A, Cho $A$, et al. High preoperative neutrophil-lymphocyte ratio predicts poor survival in patients with gastric cancer. Gastric Cancer 2010; 13(3):170-176. doi: 10.1007/ s10120-010-0554-3.

13. Gregory AD, Houghton AM. Tumor-associated neutrophils: New targets for cancer therapy. Cancer Res 2011; 71(7):2411-2416. doi: 10.1158/0008-5472.CAN-10-2583.

14. Stulting RD, Berke G. Nature of lymphocyte-tumor interaction: A general method for cellular immunoabsorption. J Exp Med 1973; 137(4):932-42. doi: 10.1084/jem.137.4.932.

15. Condeelis J, Pollard JW. Macrophages: obligate partners for tumor cell migration, invasion, and metastasis. Cell 2006; 124(2):263-266. doi: 10.1016/j.cell.2006.01.007.

16. Song Q, Wu JZ, Wang S. Low preoperative lymphocyte to monocyte ratio serves as a worse prognostic marker in patients with esophageal squamous cell carcinoma undergoing curative tumor resection. J Can 2019; 10(9):2057. doi: 10.7150 /jca.29383.

17. Yokota M, Katoh H, Nishimiya H, Kikuchi M, Kosaka Y, Sengoku N, et al. Lymphocyte-monocyte ratio significantly predicts recurrence in papillary thyroid cancer. J Surg Res 2020; 246:535-43. doi: 10.1016/j.jss.2019.09.034.

18. Zhang XK, Yang P, Zhang ZL, Hu WM, Cao Y. Preoperative low lymphocyte-to-monocyte ratio predicts poor clinical outcomes for patients with urothelial carcinoma of the upper urinary tract. Urol J 2018; 15(6):348-54. doi: 10.22037/uj.v0i0.4120.

19. Nishijima TF, Muss HB, Shachar SS, Tamura K, Takamatsu Y. Prognostic value of lymphocyte-to-monocyte ratio in patients with solid tumors: A systematic review and meta-analysis. Cancer Treat Rev 2015; 41(10):971-8. doi: 10.1016/j.ctrv.2015.10.003.

20. Li J, Cheng Y, Ji Z. Prognostic value of pretreatment lymphocyte-to-monocyte ratio in patients with urologic tumors: A PRISMA-compliant meta-analysis. Medicine (Baltimore) 2019; 98(2):e14091. doi: 10.1097/MD.0000000000014091.

21. Shigeta K, Kosaka T, Kitano S, Yasumizu Y, Miyazak Y, Mizuno R, et al. High absolute monocyte count predicts poor clinical outcome in patients with castration-resistant prostate cancer treated with docetaxel chemotherapy. Annals Surgical Oncol 2016; 23(12):4115-4122. doi: 10.1245/ s10434-016-5354-5.

22. Tang L, Li X, Wang B, Luo G, Gu L, Chen L, et al. Prognostic value of neutrophil-to-lymphocyte ratio in localized and advanced prostate cancer: A systematic review and meta-analysis. PLoS One 2016; 11(4):e0153981. doi: 10.1371/journal.pone. 0153981

23. Peng $\mathrm{H}$, Luo X. Prognostic significance of elevated pretreatment systemic inflammatory markers for patients with prostate cancer: A meta-analysis. Cancer Cell Int 2019; 19:70. doi: 10.1186/s12935-019-0785-2.

24. Han M, Partin AW, Zahurak M, Piantadosi S, Epstein Jl, Walsh PC. Biochemical (prostate specific antigen) recurrence probability following radical prostatectomy for clinically localized prostate cancer. J Urol 2003; 169(2):517-23. doi: 10.1097/01.ju.0000045749.90353.c7.

25. Karamık K, Aktaş Y, Yıldız A, Erol I, İslamoğlu E, Ateş M, et al. Predictive role of de ritis ratio in biochemical recurrence after radical prostatectomy. Medical Bulletin of Haseki/Haseki Tip Bulteni 2020; 58:84-93. DOI: 10.4274/haseki.galenos.2019.5592 